\title{
The effect of dentin pre-treatment with activated riboflavin on the bond strength of a two-step self-etch adhesive system
}

\section{Wpływ wstępnego pokrycia zębiny aktywowaną ryboflawiną na siłę wiązania dwustopniowego samotrawiącego systemu adhezyjnego}

\author{
Shahin Kasraei ${ }^{1, A, E, F}$, Maryam Mojtahedi ${ }^{2, B-D}$, Mohammad-Taghi Goodarzi ${ }^{3, A, C, E}$, Mohadese Azarsina ${ }^{4, A, D, F}$, Zahra Khamverdi ${ }^{1, B, E}$ \\ 1 Department of Restorative Dentistry, School of Dentistry, Shahid Beheshti University of Medical Sciences, Tehran, Iran \\ ${ }^{2} S c h o o l$ of Dentistry, Hamadan University of Medical Sciences, Iran \\ ${ }^{3}$ Research Center for Molecular Medicine, Hamadan University of Medical Sciences, Iran \\ ${ }^{4}$ Department of Operative Dentistry, School of Dentistry, Shahid Beheshti University of Medical Sciences, Tehran, Iran \\ A - research concept and design; $\mathrm{B}$ - collection and/or assembly of data; $\mathrm{C}$ - data analysis and interpretation; \\ $D$ - writing the article; $E$ - critical revision of the article; $F$ - final approval of the article
}

Address for correspondence

Zahra Khamverdi

E-mail: zkhamverdi@yahoo.ca

Funding sources

None declared

Conflict of interest

None declared

\section{Acknowledgements}

The authors thank the Dental Research Center and ViceChancellor of Research, Hamadan University of Medical Sciences, Iran for the financial support of this study.

Received on May 8, 2018

Reviewed on July 31, 2018

Accepted on March 6, 2019

Published online on June 28, 2019

Cite as

Kasraei S, Mojtahedi M, Goodarzi MT, Azarsina M, Khamverdi Z. The effect of dentin pre-treatment with activated riboflavin on the bond strength of a two-step self-etch adhesive system. DentMed Probl. 2019;56(2):143-148. doi:10.17219/dmp/105408

DOI

10.17219/dmp/105408

Copyright

(C) 2019 by Wroclaw Medical University

This is an article distributed under the terms of the

Creative Commons Attribution 3.0 Unported License (CC BY 3.0)

(https://creativecommons.org/licenses/by/3.0/)

\begin{abstract}
Background. The cross-linking of collagen fibers in the hybrid layer has been suggested as a way to create more durable bonds.

Objectives. This study evaluated the effect of visible light-activated riboflavin (RF) as a cross-linking agent on the durability of the dentin microtensile bond strength ( $\mathrm{TTBS}$ ) in a 2-step self-etch (SE) adhesive system.

Material and methods. The occlusal surfaces of 21 human premolar teeth were ground down to expose the dentin, and were then randomly divided into 3 groups. The $\mathrm{Clearfi}^{\circledR} \mathrm{SE}$ Bond was used in the control group. In the RF/BL group, a $0.1 \mathrm{wt} \%$ aqueous solution of RF was applied to the dentin surface before applying the adhesive and irradiating with blue light (BL) for $2 \mathrm{~min}$. In the $3^{\text {rd }}$ group, the RF-P/BL group, the RF powder was added to the adhesive primer (P) at a concentration of $0.1 \mathrm{wt} \%$. The teeth were built up using composite resin. After thermocycling, 14 resin-dentin beams from each group were prepared and stored in water for 3 months. The $\mu \mathrm{TBS}$ was determined and the data was analyzed using a linear model with a generalized estimating equation (GEE) $(p=0.05)$.

Results. The highest and the lowest $\mu \mathrm{TBS}$ belonged to the control group ( $41.15 \pm 3.50 \mathrm{MPa}$ ) and the RF-P/BL group (19.84 $\pm 3.80 \mathrm{MPa})$, respectively. The mean $\mu \mathrm{TBS}$ in the control group was significantly higher than in the RF/BL and RF-P/BL groups $(p<0.001)$, but no significant difference was found between the RF/BL and RF-P/BL groups $(p=0.598)$.

Conclusions. Pre-treatment of dentin surfaces with RF activated with BL had a negative impact on the $\mu T B S$ of the Clearfil SE Bond as a 2-step SE adhesive.
\end{abstract}

Key words: collagen, riboflavin, cross-linking agents, dentin bonding agents, photoactivation Słowa kluczowe: kolagen, ryboflawina, środki sieciujące, zębinowe środki wiążące, fotoaktywacja 


\section{Introduction}

The disintegration of an adhesive interface over time is an important factor influencing the longevity of adhesive restorations. ${ }^{1}$ Unstable polymers within the hybrid layer may cause collagen fibers to be denuded. These fibers are highly susceptible to hydrolytic and mechanical fatigue as well as degradation via the collagenolytic activity of hostderived proteases. ${ }^{2}$

Etch and rinse $(E \& R)^{3}$ and self-etch $(\mathrm{SE})^{4}$ adhesives activate dentin matrix metalloproteinases (MMPs) and may be responsible - at least in part - for the gelatinolytic activity observed in the hybrid layer. ${ }^{5,6}$ Chlorhexidine has been suggested as a strong MMP inhibitor which has antibacterial properties and, when incorporated into SE adhesives, increases the long-term durability of the adhesive without jeopardizing its mechanical properties and bond strength. ${ }^{7,8}$ Matrix metalloproteinases do not undergo turnover in dentin. Thus, inactivating these organic enzymes through the use of cross-linking agents may increase durability and may be even more effective than using MMP inhibitors like chlorhexidine. ${ }^{9,10}$

Increasing the cross-linking of collagen can be done by chemical or physical (photo-oxidative) means. The chemical approach includes the use of different types of crosslinking solutions, while the physical (photo-oxidative) approach is comprised of light exposure - particularly ultraviolet radiation. ${ }^{9,11}$ The covalent cross-links created by the external cross-linkers are very stable and deactivate the active sites of dentin proteases by decreasing the molecular mobility of the active sites or by converting the negatively-charged ionized carboxyl groups into positivelycharged amides. ${ }^{12}$ Moreover, the cross-linking technique has been suggested as a way to improve collagen's stability and resistance to degradation within the demineralized dentin matrix. ${ }^{13-15}$ Increasing the cross-linking of collagen fibrils before applying an adhesive agent may increase bonding durability. ${ }^{2,16}$ Despite the promising results of the application of cross-linkers, there are still some problems which need to be solved before they can be extensively used in clinical situations. For instance, glutaraldehyde is effective ${ }^{13}$ as a collagen cross-linker, but is also toxic. ${ }^{12}$ Grape seed extract is also effective ${ }^{10}$ and may increase the immediate bond strength to dentin. ${ }^{17}$ However, it causes a brown discoloration in dentin and the long-term durability of the resultant bond has yet to be determined. ${ }^{12}$

Riboflavin (vitamin B2; RF) is a strong free-radical producer under light activation with peak absorbance at 270 $\mathrm{nm}, 366 \mathrm{~nm}$ and $445 \mathrm{~nm} .{ }^{18}$ It significantly increases the cross-linking of type I collagen. ${ }^{19}$ When RF is photoactivated with ultraviolet A light (UVA), it produces free radicals and reactive oxygen species (ROS) such as superoxide anion $\left(\mathrm{O}_{2}{ }^{-}\right)$and hydroxyl radical $\left({ }^{\circ} \mathrm{OH}\right) .^{20}$

Some studies have shown that in E\&R adhesive systems, cross-linking dentin collagen with the use of RF/UVA increases the immediate bond strength, decreases nanole- akage, ${ }^{9,16,21}$ stabilizes the adhesive interface, and inhibits the activity of dentin MMPs. ${ }^{9,22}$ Furthermore, using RF to change the dentin collagen matrix stabilizes the fibrillar collagen network, promotes resin infiltration and hybrid layer formation ${ }^{23}$ and improves the mechanical properties and mechanical stability of a demineralized dentin substrate against hydrolytic or collagenolytic degradation. ${ }^{21,23-25}$ It has been reported that visible blue lightactivated riboflavin ( $\mathrm{RF} / \mathrm{BL}$ ) enhances the resistance to biodegradation and improves the mechanical properties of dentin collagen, bond strength, durability, and interface integrity after short-term water storage. ${ }^{26}$ Based on the literature, some protocols of RF application have succeeded in increasing microtensile bond strength ( $\mu \mathrm{TBS})$, while others demonstrated bond strength similar to or lower than that of the control group. ${ }^{21,23,25,26}$

Some previous studies have demonstrated that the acidic resin components present in $E \& R^{3}$ and $\mathrm{SE}^{4}$ adhesives increase the collagenolytic and gelatinolytic activity of a completely or partially demineralized collagen matrix. Moreover, it has been reported that SE adhesives upregulate MMP-2 synthesis by human odontoblasts ${ }^{27}$ and mild acidic resin monomers can activate MMPs by inhibiting the tissue inhibitor of metalloproteinase- 1 in TIMP-MMP complexes. ${ }^{28}$ Alternatively, these acidic resin monomers may activate latent forms of MMPs (proMMPs) via a cysteine-switch mechanism. ${ }^{29}$

When choosing a cross-linker or a photoactivation method, some clinical and safety aspects should be considered. The blue light (BL) produced by the highly popular conventional halogen-tungsten curing units (QTH) can be a good alternative to UVA light. ${ }^{26}$ Also, the need for a separate curing device (UVA) or for a separate curing process of the cross-linker using BL for optimal results $^{9,26}$ demands further investigation in order to achieve a clinically acceptable and applicable technique.

This study sought to assess the in vitro effect of visible light-activated RF on the $\mu$ TBS of a two-step SE dentin adhesive after thermocycling and 3 months of water storage. The null hypothesis was that visible light-activated RF - whether separately or incorporated into the adhesive - would have no effect on the $\mu$ TBS of a two-step SE adhesive system after thermocycling and 3 months of water storage.

\section{Material and methods}

\section{Sample preparation}

This experimental study was conducted on 21 sound human premolar teeth extracted in orthodontic treatment. We obtained verbal consent from the patients to use their extracted teeth in this in vitro study. The research protocol was approved by the institutional ethics committee of the Vice-Chancellor of Research, Hamadan 
University of Medical Sciences, Iran (protocol No. 201516p 372). This research was conducted in full accordance with the Declaration of Helsinki.

The teeth were stored in a $0.2 \%$ thymol solution until 1 week prior to the start of testing, when the teeth were stored in distilled water. The occlusal enamel of the teeth was removed using a trimmer apparatus with water cooling to expose an area of dentin approx. $5 \mathrm{~mm}$ in diameter. The surfaces of the specimens were polished with moist 600grit silicon carbide papers (Matador ${ }^{\circledR}$ 991A Soflex; Starcke $\mathrm{GmbH} \&$ Co., Melle, Germany) and the specimens were randomly divided into 3 groups of 7 in each group.

In the control group, Clearfil ${ }^{\circledR}$ SE Bond (Kuraray Medical Inc., Okayama, Japan) was applied as a two-step SE adhesive system to the prepared dentin surfaces, according to the manufacturer's instructions.

In the RF/BL group, based on previous studies, a $0.1 \mathrm{wt} \%$ RF solution was prepared by dissolving $100 \mathrm{mg}$ of riboflavin-5-phosphate powder (Sigma-Aldrich, St. Louis, USA) into $100 \mathrm{~mL}$ of distilled water and then adjusting the $\mathrm{pH}$ of the RF solution to $\mathrm{pH}=3 .{ }^{19,28}$ The prepared $0.1 \mathrm{wt} \%$ RF solutions were stored in lightproof test tubes at room temperature $\left(24^{\circ} \mathrm{C}\right)$ in order to prevent light activation. Using a sampler, $15 \mu \mathrm{L}$ of the RF solution was applied to the dentin surfaces. The treated dentin surfaces were gently air-dried and photoactivated using the BL generated by QTH light curing unit (Optilux ${ }^{\circledR} 501$; Demetron Kerr, Danbury, USA) with an output light spectrum of 375$520 \mathrm{~nm}$, a minimum light intensity of $600 \mathrm{~mW} / \mathrm{cm}^{2}$ and a light guide diameter of $8 \mathrm{~mm}$ for $2 \mathrm{~min}$. The tip of the light guide was placed as close to the surface as possible. Clearfil SE primer and bonding agent were then applied to the prepared dentin surface and cured according to the manufacturer's instructions.

In the RF-P/BL group, a riboflavin-primer mixture (RF-P) was created by dissolving RF powder in the primer until reaching a concentration of $0.1 \mathrm{wt} \%$. The dentin surface was treated with the RF-P for $20 \mathrm{~s}$, air-dried for $5 \mathrm{~s}$ and photoactivated with BL for $2 \mathrm{~min}$. Then, Clearfil SE Bond adhesive was applied and cured for $10 \mathrm{~s}$.

All of the teeth were built up by using $4 \mathrm{~mm}$ of composite resin (Filtek ${ }^{\circledR}$ Z250; 3M ESPE, St. Paul, USA) in 1-millimeter increments. After being stored for $24 \mathrm{~h}$ in distilled water at $37^{\circ} \mathrm{C}$, the prepared teeth were subjected to 5,000 thermal cycles $\left(5-55^{\circ} \mathrm{C}, 15 \mathrm{~s}\right.$ dwell time). To assess the $\mu \mathrm{TBS}$, the teeth were then sectioned using a low-speed diamond saw with water cooling in order to obtain resin-dentin beams measuring $1 \times 1 \mathrm{~mm}$. Two resin-dentin beams were selected from the middle part of each specimen. Thus, each group had 14 specimens. The beams were then subjected to 3 months of water storage at $37^{\circ} \mathrm{C}$.

\section{Bond strength evaluation}

Using cyanoacrylate glue, the beams were mounted on a custom-made metal jig in a universal testing machine
(STM-20; Santam, Tehran, Iran). The $\mu$ TBS was measured with a $50 \mathrm{~N}$ load cell at a crosshead speed of $1 \mathrm{~mm} / \mathrm{min}$.

\section{Failure mode evaluation}

All the fractured samples were evaluated twice under a stereomicroscope (SZ40; Olympus, Tokyo, Japan) at $\times 40$ magnification to determine the mode of failure (adhesive, cohesive in dentin, cohesive in composite resin, and mixed) by 1 operator.

\section{Sodium dodecyl sulfate - polyacrylamide gel electrophoresis analysis}

The cross-linking effect of the prepared RF solutions on type I collagen was evaluated with sodium dodecyl sulfate polyacrylamide gel electrophoresis (SDS-PAGE). Collagen mixed with distilled water served as the negative control group and $0.1 \%$ RF/UVA $(\mathrm{pH}=7)$ was used as the positive control. The samples were diluted with the sample buffer and subjected to SDS-PAGE (8\%) at $150 \mathrm{~V}$; the gel was stained with Coomassie blue and then photographed.

\section{Micromorphological assessment of the resin-dentin interface using scanning electron microscopy}

Two extra resin-dentin beams from each group were selected and prepared for micromorphological assessment of the resin-dentin interface using scanning electron microscope (SEM). These beams were mounted into acrylic resin. The mounted beams were polished with 600-, 800-, 1200-, 1500- and 3500-grit silicon carbide papers (Matador 991A Soflex; Starcke GmbH \& Co KG). The specimens were then etched with $35 \%$ phosphoric acid for $20 \mathrm{~s}$, rinsed for $20 \mathrm{~s}$ and dried with oil-free compressed air. The beams were immersed in a $5.25 \%$ sodium hypochlorite solution for $20 \mathrm{~min}$ and were thoroughly rinsed under running water for $5 \mathrm{~min}$. The specimens were then dehydrated using increasing concentrations of ethanol - i.e., $33 \%, 50 \%, 70 \%$, and $85 \%$ - for $15 \mathrm{~min}$ each, followed by $90 \%, 95 \%$ and $100 \%$ ethanol for 10 min each. After drying, the beams were mounted and sputter-coated with a goldpalladium alloy for $180 \mathrm{~s}$ (Sputter Coater SC 7620; Quorum Tech, Lewes, UK). The specimens were then evaluated under SEM (LEO 1450VP; Carl Zeiss, Oberkochen, Germany) at $\times 5,000$ magnification.

\section{Statistical analysis}

The results of $\mu$ TBS testing were analyzed with one-way analysis of variance (ANOVA) for the "dentin pretreatment" factor (without RF, with RF/BL or with RF-P/BL), followed by Tukey's post-hoc pairwise comparison tests. Statistical significance was set at $p<0.05$. 


\section{Results}

In all columns, bands were seen at about $130 \mathrm{KDa}$, belonging to $\alpha 1$ and $\alpha 2$ monomers; in all groups, apart from the negative control group, well residuals were also seen on top of the stacking gel (Fig. 1).

The highest and the lowest bond strength belonged to the control group (41.15 \pm 3.50$)$ and the RF-P/BL group (19.84 \pm 3.80 ), respectively. The generalized estimating equation (GEE) model revealed that the mean $\mu \mathrm{TBS}$ in the control groupwassignificantlyhigher than in the RF/BLandRF-P/BL groups $(p<0.001)$; however, the difference in the mean $\mu \mathrm{TBS}$ between the RF/BL and the RF-P/BL groups was not statistically significant $(p=0.598)$. The mean bond strength values and the frequency distribution of the mode of failure in the study groups are shown in Table 1.

Figure 2 shows the SEM images of the resin-dentin interface in selected samples from different groups after 5,000 thermal cycles and 3 months of water storage. A uniform hybrid layer and long resin tags with relatively regular distribution and straight orientation were clearly seen in the control group (without RF). However, in the $\mathrm{RF} / \mathrm{BL}$ group, a hybrid layer was formed in only a few sites and there were only a few very fine resin tags with irregular distribution. In the RF-P/BL group, the hybrid layer was less uniform. The resin tags varied in height and showed a more irregular orientation compared to the control group (Fig. 2).

\section{Discussion}

In SDS-PAGE analysis, both the RF/BL and RF-P/BL groups showed stronger $\alpha$-bonds and fewer fragments than the control group, which indicates the formation

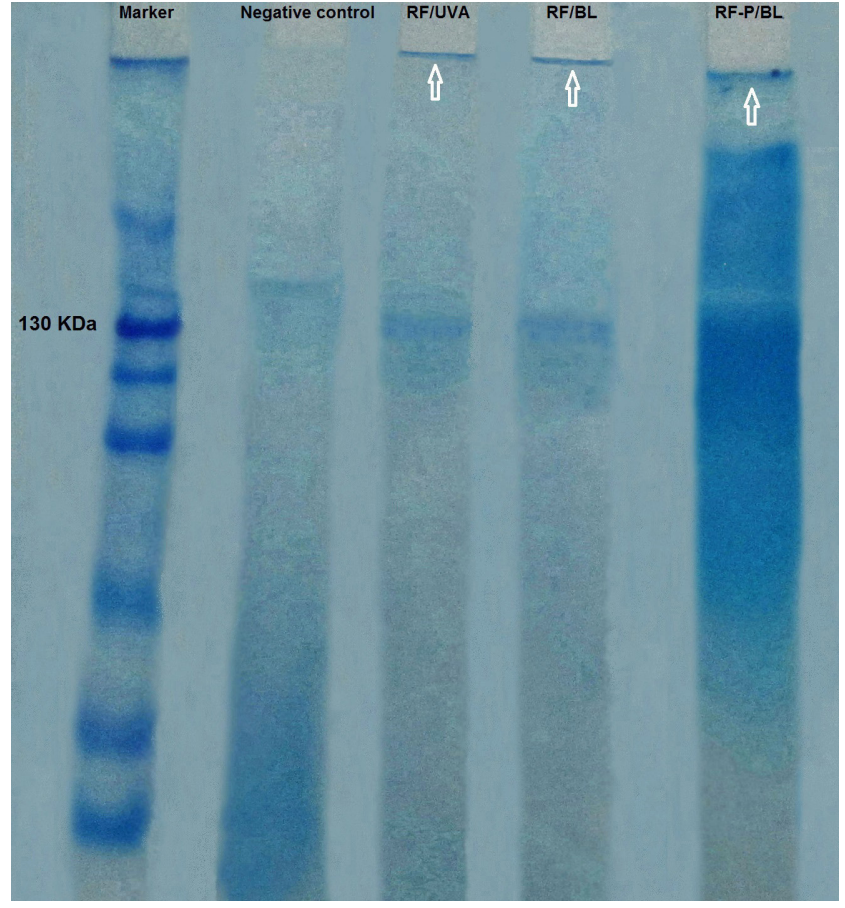

Fig. 2. Selected SEM images indicating the resin-dentin interface (at $\times 5,000$ magnification)

A - control group; $B$ - RF/BL group; C - RF-P/BL group.

of intermolecular cross-links (Fig. 1). There were insoluble residuals on the wells in both groups that were identified as giant cross-linked collagen molecules. This finding was in line with the results of Chiang et al. ${ }^{21}$

The $\mu$ TBS measured in the group without RF in our study (41.15 MPa) was similar to the value reported in previous studies using a similar test ${ }^{30,31}$ and the failure mode was mostly cohesive in the composite resin. The $\mu$ TBS value in the presence of RF alone $(p<0.001)$ or in combination with the primer $(p<0.001)$ was lower than that in the control
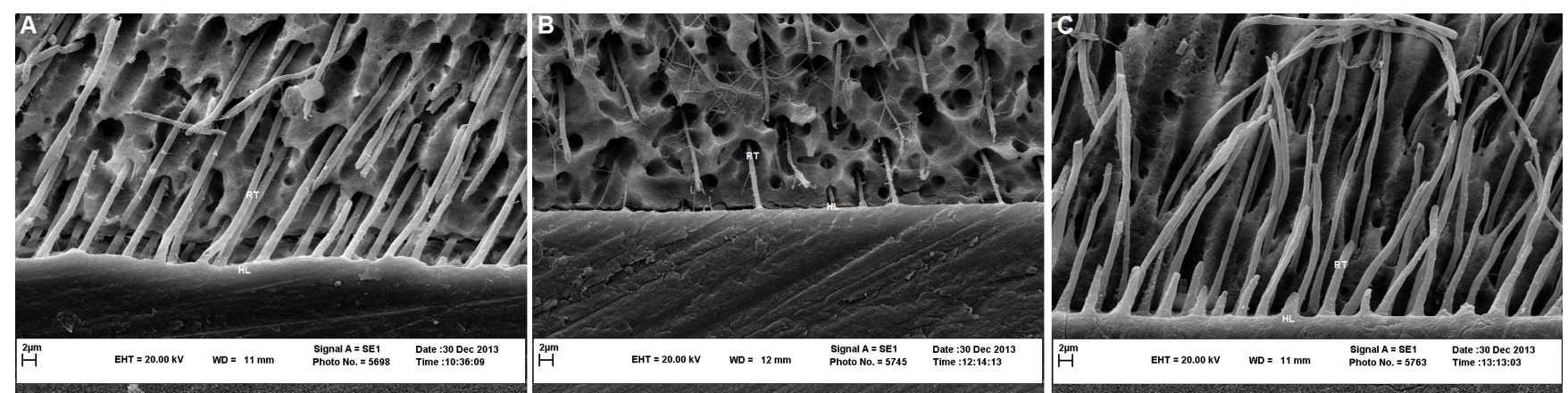

Fig. 1. Results of SDS-PAGE analysis. The arrows indicate high-molecular-weight residuals on top of the stacking gel

Table 1. Mean value (SD) of bond strength (in MPa) and failure modes of $\mu$ TBS test

\begin{tabular}{|l|c|c|c}
\multicolumn{1}{c|}{ Group } & Surface pretreatment & Mean of microtensile bond strength & Failure mode (A/M/CR/CD) \\
\hline Control & no pretreatment & $41.15(3.50) \mathrm{a}$ & $0 / 5 / 7 / 2$ \\
RF/BL & application and photoactivation of RF\% $0.1(\mathrm{pH}=3)$ & $20.79(4.41) \mathrm{b}$ & $2 / 12 / 0 / 0$ \\
RF-P/BL & application and photoactivation of riboflavin-primer mixture & $19.84(3.80) \mathrm{b}$ & $2 / 12 / 0 / 0$ \\
\hline
\end{tabular}

SD - standard deviation; RF - riboflavin; RF-P - riboflavin-primer mixture; BL - blue light; A - adhesive; $M$ - mixed; $C R$ - cohesive in resin composite;

$\mathrm{CD}$ - cohesive in dentin. Values with different uppercase letters indicate significant differences according to Tukey's test ( $p<0.05)$. 
group; therefore, the null hypothesis of the study was upheld. No significant difference was found in the $\mu$ TBS reduction in the understudy specimens between the 2 modes of application for the cross-linkers $(\mathrm{p}=0.598)$. The majority of failures observed in these 2 groups were mixed.

One advantage of inactivating proteolytic enzymes in a dentin matrix by a cross-linking agent is its non-specific mechanism, since it cross-links all the MMPs present in dentin and collagen fibers. These cross-links are composed of covalent bonds stabilized over time. ${ }^{9}$ Riboflavin is a nontoxic vitamin used as a food coloring agent. ${ }^{9}$ Moreover, its light yellow color makes it more suitable for dental purposes than other photosensitizing chromophores, such as porphyrins, rose bengal and methylene blue. ${ }^{21}$

In contrast to our findings, some studies have reported that dentin surface pretreatment with UVA-activated RF increased $\mu$ TBS immediately and after storage in comparison with a control group. ${ }^{9,16,21,32}$ The difference between our results and those of previous studies may be attributed to the duration of exposure and the source of light used for activation, as well as the storage conditions.

As with our study, some previous investigations have reported the inefficacy of RF or even its adverse effects on bond strength. ${ }^{21,26}$ Fawzy et al. reported that using 1\% RF and single-step photoactivation with BL simultaneously with the bonding produced lower $\mu$ TBS after $24 \mathrm{~h}$ and after 4 months of water storage compared to the control group. ${ }^{26}$ Cova et al. reported that when comparing immediate and delayed bond strength, all specimens crosslinked with RF still demonstrated some level of reduction in the mean $\mu$ TBS after 12 months. ${ }^{9}$ It should be noted that all of these studies were conducted on E\&R adhesives and did not evaluate SE adhesives.

A reduction in bond strength when RF is used may be due to the strengthening effect of hydration by RF/UVA. ${ }^{33}$ The increased hydration of activated RF may be unfavorable to the formation of a stable hybrid layer. ${ }^{21}$ Riboflavin can compromise the function of the adhesive system by increasing water sorption from dentin, diluting the primer and maintaining the water content of the primer.

A residual weak collagen matrix within the hybrid layer may be another possible reason for the reduced $\mu$ TBS in specimens treated with RF after thermocycling and water storage found in our study. As with non-specific inhibitors, the main drawback of cross-linkers used to inactivate MMPs is that they leave a water-rich, resin-deficient collagen matrix with poor mechanical properties within the hybrid layer. ${ }^{2,34}$ These very fragile collagen fibrils are susceptible to creep and subsequent fatigue rupture after long-term functioning. ${ }^{17}$

It should be noted that in this study, in contrast to the E\&R systems used in previous studies, a SE adhesive system was used. In the process of SE adhesive system application, the smear layer containing proteins, collagen and debris is not eliminated from the dentin surface by the application of acidic monomers. Thus, applying activated RF can cross- link the residual proteins and collagens in the smear layer and cover the dentin surface during the application of acidic monomers of the SE adhesive, obstructing the path of the resin, hindering its penetration into the spaces between collagen fibers. The observation of a partially or deficiently formed hybrid layer and sparse fine resin tags on the SEM imagery from the RF/BL group can confirm this theory. Collagen fibrils which are not fully covered by the resin monomers during the bonding process are susceptible to mechanical fatigue as well as hydrolytic and enzymatic degradation by collagenolytic enzymes. ${ }^{35}$

In the RF-P/BL group, a hybrid layer was formed and the length and distribution of resin tags was less uniform than in the control group. However, due to the simultaneous use of RF and primer, the inhibition of resin penetration was not observed in the respective SEM imagery. In this group, RF was incorporated into the Clearfil SE Bond primer until a $0.1 \%$ concentration was reached, and after RF-P was applied to the dentin surface, light activation was carried out for $2 \mathrm{~min}$. It has been reported that treating dentin powder with Clearfil SE Bond primer for 2 min instead of $20 \mathrm{~s}$ not only increases collagenolytic activity, but also disables enzymatic inhibition to the point where only $2 \%$ chlorhexidine $(\mathrm{CHX})$ can still demonstrate a significant inhibitory effect. ${ }^{36}$ Therefore, the reduction in mean $\mu$ TBS observed in RF-P/BL group may be due to the long duration of dentin treatment with Clearfil SE Bond primer before the application of the bonding agent or due to the low concentration of RF used. Further studies are required to assess the surface micromorphology of treated dentin and to find the best concentration of RF in the SE primer.

The likely negative effect of directly incorporating RF into an SE adhesive primer on the degree of conversion and the mechanical characteristics of a polymerized resin may be another reason for decreased bond strength. This process negatively affects the mechanical properties of polymerized resins. For instance, it has been reported that the addition of $1 \% \mathrm{CHX}$ to various resin mixtures with different hydrophilicities leads to a reduction in the modulus of elasticity (MOE) of polymerized resins by $27-48 \% .{ }^{37}$ When directly incorporating RF into the primer composition, the possibility that it may react with the primer components and interfere with its optimal functioning cannot be ignored. However, further tests and chemical analyses are required in order to suggest this method with confidence.

In the present study, the bond strength of the RF/BL and RF-P/BL groups was similar. Despite the vastly different microscopic images, different potential mechanisms involved in reducing the bond strength in the 2 groups may explain this similarity. As discussed earlier, the obstruction of the path of resin penetration into the spaces between collagen fibers as a result of collagen cross-linking in the smear layer may be responsible, at least in part, for the reduction in bond strength in the RF/BL group; in the RF-P/BL group, however, the reduction in bond strength may be due to the long dura- 
tion of dentin treatment with Clearfil SE Bond primer before applying the bonding agent, the low concentration of RF used or the likely negative impact of directly incorporating $\mathrm{RF}$ into the $\mathrm{SE}$ adhesive primer formulation.

\section{Conclusions}

Within the limitations of this study, cross-linking of collagen type I fibers did occur as the result of the $0.1 \mathrm{wt} \%$ aqueous solution of RF and the QTH light activation for 2 min. Pretreatment of the dentin surface with QTH light-activated RF decreased the $\mu$ TBS of the Clearfil SE Bond as a SE adhesive system. No difference was detected between the application of $0.1 \mathrm{wt} \% \mathrm{RF}$ alone to the dentin surface and the incorporation of $0.1 \%$ wt RF into the primer of the two-step SE adhesive system.

\section{ORCID iDs}

Shahin Kasraei (1) https://orcid.org/0000-0003-0167-4704

Maryam Mojtahedi (1) https://orcid.org/0000-0002-7885-1847

Mohammad-Taghi Goodarzi (1) https://orcid.org/0000-0002-5546-5812

Mohadese Azarsina (1) https://orcid.org/0000-0001-6190-7472

Zahra Khamverdi (i) https://orcid.org/0000-0001-9623-5573

\section{References}

1. De Munck J, Van Landuyt $K$, Peumans $M$, et al. A critical review of the durability of adhesion to tooth tissue: Methods and results. J Dent Res. 2005;84(2):118-132.

2. Perdigão J, Reis A, Loguercio AD. Dentin adhesion and MMPs: A comprehensive review. J Esthet Restor Dent. 2013;25(4):219-241.

3. Mazzoni A, Pashley DH, Nishitani Y, et al. Reactivation of inactivated endogenous proteolytic activities in phosphoric acid-etched dentine by etch-and-rinse adhesives. Biomaterials. 2006;27(25):4470-4476.

4. Nishitani Y, Yoshiyama M, Wadgaonkar B, et al. Activation of gelatinolytic/collagenolytic activity in dentin by self-etching adhesives. Eur J Oral Sci. 2006;114(2):160-166.

5. Mazzoni A, Carrilho M, Papa V, et al. MMP-2 assay within the hybrid layer created by a two-step etch-and-rinse adhesive: Biochemical and immunohistochemical analysis. J Dent. 2011;39(7):470-477.

6. Mazzoni A, Nascimento FD, Carrilho M, et al. MMP activity in the hybrid layer detected with in situ zymography. J Dent Res. 2012;91(5):467-472.

7. Hiraishi N, Yiu CK, King NM, Tay FR. Effect of chlorhexidine incorporation into a self-etching primer on dentine bond strength of a luting cement. J Dent. 2010;38(6):496-502.

8. Stanislawczuk R, Pereira F, Muñoz MA, et al. Effects of chlorhexidine-containing adhesives on the durability of resin-dentine interfaces. J Dent. 2014;42(1):39-47.

9. Cova A, Breschi L, Nato F, et al. Effect of UVA-activated riboflavin on dentin bonding. J Dent Res. 2011;90(12):1439-1445.

10. Frassetto A, Breschi L, Turco G, et al. Mechanisms of degradation of the hybrid layer in adhesive dentistry and therapeutic agents to improve bond durability - a literature review. Dent Mater. 2016;32(2):e41-e53.

11. Seseogullari-Dirihan R, Tjäderhane L, Pashley DH, Tezvergil-Mutluay A. Effect of ultraviolet A-induced crosslinking on dentin collagen matrix. Dent Mater. 2015;31(10):1225-1231.

12. Tjäderhane $L$, Nascimento FD, Breschi $L$, et al. Strategies to prevent hydrolytic degradation of the hybrid layer - a review. Dent Mater. 2013;29(10):999-1011.

13. Bedran-Russo AK, Pashley DH, Agee K, Drummond JL, Miescke KJ. Changes in stiffness of demineralized dentin following application of collagen crosslinkers. J Biomed Mater Res B Appl Biomater. 2008;86(2):330-334.

14. Walter R, Miguez PA, Arnold RR, Pereira PN, Duarte WR, Yamauchi M. Effects of natural cross-linkers on the stability of dentin collagen and the inhibition of root caries in vitro. Caries Res. 2008;42(4):263-268.
15. Xu C, Wang Y. Cross-linked demineralized dentin maintains its mechanical stability when challenged by bacterial collagenase. J Biomed Mater Res B Appl Biomater. 2010;96(2):242-248.

16. Daood U, Swee Heng C, Neo Chiew Lian J, Fawzy AS. In vitro analysis of riboflavin-modified, experimental, two-step etch-and-rinse dentin adhesive: Fourier transform infrared spectroscopy and micro-Raman studies. Int J Oral Sci. 2015;7(2):110-124.

17. Fang $M$, Liu R, Xiao $Y$, et al. Biomodification to dentin by a natural crosslinker improved the resin-dentin bonds. J Dent. 2012;40(6):458-466.

18. Spoerl E, Mrochen M, Sliney D, Trokel S, Seiler T. Safety of UVA-riboflavin cross-linking of the cornea. Cornea. 2007;26(4):385-389.

19. Arbelaez MC, Sekito MB, Vidal C, Choudhury SR. Collagen crosslinking with riboflavin and ultraviolet-A light in keratoconus: One-year results. Oman J Ophthalmol. 2009;2(1):33-38.

20. McCall AS, Kraft S, Edelhauser HF, et al. Mechanisms of corneal tissue cross-linking in response to treatment with topical riboflavin and long-wavelength ultraviolet radiation (UVA). Invest Ophthalmol Vis Sci. 2010;51(1):129-138.

21. Chiang YS, Chen YL, Chuang SF, et al. Riboflavin-ultraviolet-A-induced collagen cross-linking treatments in improving dentin bonding. Dent Mater. 2013;29(6):682-692.

22. Seseogullari-Dirihan R, Apollonio F, Mazzoni A, et al. Use of crosslinkers to inactivate dentin MMPs. Dent Mater. 2016;32(3):423-432.

23. Daood U, lqbal K, Nitisusanta LI, Fawzy AS. Effect of chitosan/riboflavin modification on resin/dentin interface: Spectroscopic and microscopic investigations. J Biomed Mater Res A. 2013;101(7):1846-1856.

24. Fawzy A, Nitisusanta L, lqbal K, Daood U, Beng LT, Neo J. Characterization of riboflavin-modified dentin collagen matrix. J Dent Res. 2012;91(11):1049-1054.

25. Fawzy AS, Nitisusanta LI, Iqbal K, Daood U, Beng LT, Neo J. Chitosan/riboflavin-modified demineralized dentin as a potential substrate for bonding. J Mech Behav Biomed Mater. 2013;17:278-289.

26. Fawzy AS, Nitisusanta LI, Iqbal K, Daood U, Neo J. Riboflavin as a dentin crosslinking agent: Ultraviolet A versus blue light. Dent Mater. 2012;28(12):1284-1291.

27. Lehmann N, Debret $R$, Roméas $A$, et al. Self-etching increases matrix metalloproteinase expression in the dentin-pulp complex. J Dent Res. 2009;88(1):77-82.

28. Ishiguro K, Yamashita K, Nakagaki H, Iwata K, Hayakawa T. Identification of tissue inhibitor of metalloproteinases-1 (TIMP-1) in human teeth and its distribution in cementum and dentine. Arch Oral Biol. 1994;39(4):345-349.

29. Tallant C, Marrero A, Gomis-Rüth FX. Matrix metalloproteinases: Fold and function of their catalytic domains. Biochim Biophys Acta. 2010;1803(1):20-28.

30. Reis A, Loguercio AD, Manso AP, et al. Microtensile bond strengths for six 2-step and two 1-step self-etch adhesive systems to enamel and dentin. Am J Dent. 2013;26(1):44-50.

31. Scherrer SS, Cesar PF, Swain MV. Direct comparison of the bond strength results of the different test methods: A critical literature review. Dent Mater. 2010;26(2):e78-e93.

32. Hass V, Luque-Martinez IV, Gutierrez MF, et al. Collagen cross-linkers on dentin bonding: Stability of the adhesive interfaces, degree of conversion of the adhesive, cytotoxicity and in situ MMP inhibition. Dent Mater. 2016;32(6):732-741.

33. Hayes S, Boote C, Kamma-Lorger CS, et al. Riboflavin/UVA collagen cross-linking-induced changes in normal and keratoconus corneal stroma. PLoS One. 2011;6(8):e22405.

34. Liu $Y$, Tjäderhane $L$, Breschi $L$, et al. Limitations in bonding to dentin and experimental strategies to prevent bond degradation. J Dent Res. 2011;90(8):953-968.

35. Pashley DH, Tay FR, Yiu C, Breschi L, et al. Collagen degradation by host-derived enzymes during aging. J Dent Res. 2004;83(3):216-221.

36. Zhou J, Tan J, Yang X, Xu X, Li D, Chen L. MMP-inhibitory effect of chlorhexidine applied in a self-etching adhesive. J Adhes Dent. 2011;13(2):111-115

37. Cadenaro $M$, Breschi $L$, Rueggeberg FA, et al. Effect of adhesive hydrophilicity and curing time on the permeability of resins bonded to water vs ethanol-saturated acid-etched dentin. Dent Mater. 2009;25(1):39-47. 